\begin{tabular}{|c|c|}
\hline Annals of Pure and Applied Mathematics & \\
\hline Vol. 20, No. 1, 2019, 21-24 & $\overline{\text { Ann }}$ \\
\hline ISSN: 2279-087X (P), 2279-0888(online) & \\
\hline Published on 6 August 2019 & \\
\hline Www.researchmathsci.org & \\
\hline DOI: http://dx.doi.org/10.22457/apam.625v20n1a4 & \\
\hline
\end{tabular}

\title{
Chebychev Subspaces of Orlicz Function Space
}

\author{
G. Ghawadrah
}

\author{
Department of Mathematics, An-Najah National University \\ Nablus, Palestine. E-mail: g.ghawadrah@najah.edu \\ Received 7 July 2019; accepted 31 July 2019
}

\begin{abstract}
In this paper it is proved that $G$ is a Chebychev subspace of a Banach space $X$ if and only if $L^{\phi}(\mu, G)$ is a Chebyshev subspace of $L^{\phi}(\mu, X)$, where $L^{\phi}(\mu, X)$ is an Orlicz function space with Luxemburg norm.
\end{abstract}

Keywords: Proximinally additive, Chebychev space, Orlicz space.

\section{AMS Mathematics Subject Classification (2010): 46E30}

\section{Introduction}

Let $\phi$ be an Orlicz function on $[0, \infty)$ (i.e. a continuous, strictly increasing convex function satisfying $\phi(0)=0$ and $\left.\lim _{t \rightarrow \infty} \phi(t)=\infty\right)$. Let $(\Omega, \mu)$ be a finite measure, for a real Banach space $X$, The orlicz space $L^{\phi}(\mu, X)$ is a space of all measurable functions $f: \Omega \rightarrow X$ such that $\int_{\Omega} \phi\left(c^{-1}\|f(t)\|\right) d \mu(t)<\infty$, for some $c>0$. Define a Luxemburg norm on $L^{\phi}(\mu, X)$ by $\|f\|_{\phi}=\inf \left\{c>0: \int_{\Omega} \phi\left(c^{-1}\|f(t)\|\right) d \mu(t) \leq 1\right\}$, for more see [9].

For the subset $G$ of the normed linear space $(X,\|\|$.$) , we define, for x \in X, d(x, G)=$ $\inf \{\|x-g\|: g \in G\}$. If $G$ is a subspace of $X$, an element $g_{0} \in G$ is called a best approximant of $x$ in $G$ if $\left\|x-g_{0}\right\|=d(x, G)$. We shall denote the set of all best approximants of $x$ in $G$ as $P(x, G)$. If for each $x \in X$, the set $P(x, G) \neq \varnothing$, then $G$ is said to be proximinal in $X$, and if $P(x, G)$ is a singleton for each $x \in X$ than $G$ is called a Chebychev subspace. A subspace $G$ of a Banach space $X$ is said to be proximinally additive if $G$ is closed and $z_{1}+z_{2} \in P\left(x_{1}+\right.$ $\left.x_{2}, G\right)$ whenever $z_{1} \in P\left(x_{1}, G\right)$ and $z_{2} \in P\left(x_{2}, G\right)$. For more see [6], [7].

In this paper we prove that: $G$ is a Chebychev subspace of a Banach space $X$ if and only if $L^{\phi}(\mu, G)$ is a Chebyshev subspace of $L^{\phi}(\mu, X)$, where $L^{\phi}(\mu, X)$ is an Orlicz function space with Luxemburg norm. In [3], it was proved the same result for the linear metric space $L^{\phi}(\mu, X)$, with $\phi$ is a modulus function, and $d(f, g)=\int_{\Omega} \phi(\|f(t)-g(t)\|) d \mu(t)$ is the metric, for more results about this metric space see $[2,3,4,5]$.

Throughout this paper we take $\Omega=[0,1]$ and $\phi$ is an Orlicz function with $\phi(0)=$ $0, \phi(1)=1$.

\section{Main results}

Lemma 2.1. [3] Suppose that $G$ is a proximinal subspace of a Banach space $X$ and that $G$ is proximinally additive in $X$. Then $G$ is Chebyshev. 


\section{G. Ghawadrah}

Theorem 2.2. Let $X$ be a Banach space and $G$ be a closed subspace of $X$. if $G$ is proximinally additive in $X$, then $L^{\phi}(\mu, G)$ is proximinally additive in $L^{\phi}(\mu, X)$.

Proof: Suppose $g_{1} \in P\left(f_{1}, L^{\phi}(\mu, G)\right)$, and $g_{2} \in P\left(f_{2}, L^{\phi}(\mu, G)\right)$, then by [8, corollary 2.1] we get $g_{1}(t) \in P\left(f_{1}(t), G\right), g_{2}(t) \in P\left(f_{2}(t), G\right), \forall t$.

Hence, $\forall t \in \Omega, \forall \mathrm{y} \in \mathrm{G}$

$$
d\left(\left(f_{1}+f_{2}\right)(t), G\right)=\left\|\left(f_{1}+f_{2}\right)(t)-\left(g_{1}+g_{2}\right)(t)\right\| \leq\left\|\left(f_{1}+f_{2}\right)(t)-y\right\|
$$

Thus, $\forall t \in \Omega, \forall y \in L^{\phi}(\mu, G):\left\|\left(f_{1}+f_{2}\right)(t)-\left(g_{1}+g_{2}\right)(t)\right\| \leq\left\|\left(f_{1}+f_{2}\right)(t)-h(t)\right\|$.

Since $\phi$ is increasing, then $\forall h \in L^{\phi}(\mu, G)$ we have that

$\int_{0}^{1} \phi\left(c^{-1}\left\|\left(f_{1}+f_{2}\right)(t)-\left(g_{1}+g_{2}\right)(t)\right\|\right) d \mu(t) \leq \int_{0}^{1} \phi\left(c^{-1}\left\|\left(f_{1}+f_{2}\right)(t)-h(t)\right\| d \mu(t)\right)$

Thus,

$$
\begin{aligned}
\inf \left\{c>0: \int_{0}^{1} \phi\right. & \left.\left(c^{-1}\left\|\left(f_{1}+f_{2}\right)(t)-\left(g_{1}+g_{2}\right)(t)\right\|\right) d \mu(t)\right\} \\
& \leq \inf \left\{c>0: \int_{0}^{1} \phi\left(c^{-1}\left\|\left(f_{1}+f_{2}\right)(t) h(t)\right\| d \mu(t)\right)\right\}
\end{aligned}
$$

Therefore, $\forall h \in L^{\phi}(\mu, G),\left\|\left(f_{1}+f_{2}\right)-\left(g_{1}+g_{2}\right)\right\|_{\phi} \leq\left\|\left(f_{1}+f_{2}\right)-\square\right\|_{\phi}$ And so, $g_{1}+g_{2} \in P\left(f_{1}+f_{2}, L^{\phi}(\mu, G)\right)$. Thus, $L^{\phi}(\mu, G)$ is proximinally additive.

Theorem 2.3. Let $X$ be a Banach space and $G$ be a closed subspace of $X$. If $L^{\phi}(\mu, G)$ is proximinally additive in $L^{\phi}(\mu, X)$, then $G$ is proximinally additive in $X$.

Proof: Suppose $L^{\phi}(\mu, G)$ is proximinally additive in $L^{\phi}(\mu, X)$, and let $z_{i} \in P\left(x_{i}, G\right)$ for $i=1,2$; we want to show $z_{1}+z_{2} \in P\left(x_{1}+x_{2}, G\right)$.

Now let $f_{i}(t)=x_{i}$ and $g_{i}(t)=z_{i}, \forall t$ and for $i=1,2$.

It is clear such that $f_{i}, g_{i} \in L^{\phi}(\mu, X)$, and $g_{i} \in L^{\phi}(\mu, G), i=1,2$,

First, we show that $g_{i} \in P\left(f_{i}, L^{\phi}(\mu, G)\right), i=1,2$.

Now, for $i=1,2$, we have

$z_{i} \in P\left(x_{i}, G\right) \Longrightarrow\left\|x_{i}-z_{i}\right\| \leq\left\|x_{i}-y\right\|, \forall y \in G$

$\Longrightarrow\left\|f_{i}(t)-g_{i}(t)\right\| \leq\left\|f_{i}(t)-y\right\|, \forall y \in G, \forall t$

$\Rightarrow\left\|f_{i}(t)-g_{i}(t)\right\| \leq\left\|f_{i}(t)-h(t)\right\|, \forall t, \forall h \in L^{\phi}(\mu, G)$

Since $\phi$ is strictly increasing, then $\forall h \in L^{\phi}(\mu, G)$ we have that $\int_{0}^{1} \phi\left(c^{-1}\left\|f_{i}(t)-g_{i}(t)\right\|\right) d \mu(t) \leq \int_{0}^{1} \phi\left(c^{-1}\left\|f_{i}(t)-h(t)\right\| d \mu(t)\right)$

Hence, $\forall h \in L^{\phi}(\mu, G)$ :

$$
\begin{aligned}
\inf \left\{c>0: \int_{0}^{1} \phi\right. & \left.\left(c^{-1}\left\|f_{i}(t)-g_{i}(t)\right\|\right) d \mu(t) \leq 1\right\} \\
& \leq \inf \left\{c>0: \int_{0}^{1} \phi\left(c^{-1}\left\|f_{i}(t)-h(t)\right\|\right) d \mu(t) \leq 1\right\}
\end{aligned}
$$

Thus, $\left\|f_{i}-g_{i}\right\|_{\phi} \leq\left\|f_{i}-h\right\|, i=1,2, \forall h \in L^{\phi}(\mu, G)$.

So we get that $g_{i} \in P\left(f_{i}, L^{\phi}(\mu, G)\right), i=1,2$.

Since $L^{\phi}(\mu, G)$ is proximinally additive in $L^{\phi}(\mu, X)$, then

$$
g_{1}+g_{2} \in P\left(f_{1}+f_{2}, L^{\phi}(\mu, G)\right) \text {. }
$$




\section{Chebychev Subspaces of Orlicz Function Space}

By corollary 2.1 of [8], we have that

$$
g_{1}(t)+g_{2}(t) \in P\left(f_{1}(t)+f_{2}(t), G\right) .
$$

Hence, $z_{1}+z_{2} \in P\left(x_{1}+x_{2}, G\right)$. Therefore $G$ is proximinally additive in $X$.

Corollary 2.4. Let $X$ be a Banach space and $G$ be a closed subspace of $X . L^{\phi}(\mu, G)$ is proximinally additive in $L^{\phi}(\mu, X)$ if and only if $G$ is proximinally additive in $X$.

Theorem 2.5. Let $G$ be a closed subspace of a Banach space $X$. Then the following are equivalent :

(i) $G$ is proximinal in $X$

(ii ) $L^{\phi}(\mu, G)$ is proximinal in $L^{\phi}(\mu, X)$.

Proof: By[1, theorem 3.7] and [8, theorem 2.1] we get that:

$G$ is proximinal $\Leftrightarrow L^{l}(\mu, G)$ is proximinal $\Leftrightarrow L^{\phi}(\mu, G)$ is proximinal .

Theorem 2.6. Let $G$ be a closed subspace of a Banach space $X$ which is proximinally additive in $X$, then the following are equivalent:

(i) $G$ is a Chebyshev subspace of $X$.

(ii) $L^{\phi}(\mu, G)$ is a Chebyshev subspace of $L^{\phi}(\mu, X)$.

Proof: By theorem (2.5), and corollary 2.4

Orlicz spaces generalize $L^{p}(\mu, X)$ for $1<p<\infty$, in the sense that if $\phi(t)=t^{p}$, then $\|f\|_{\phi}=\|f\|_{p}$ and so $L^{\phi}(\mu, X)=L^{p}(\mu, X)$. Hence, the following theorem from [3] is a direct result of corollary 2.4:

Theorem 2.7. Let $G$ be a closed subspace of a Banach space $X$. Then the following are equivalent :

(i) $G$ is proximinally additive in $X$.

(ii) $L^{p}(\mu, G)$ is proximinally additive in $L^{p}(\mu, X), 1<p<\infty$.

\section{Conclusion}

If $G$ is a closed subspace of a Banach space $X$, then we get that: $G$ is a Chebychev subspace of a Banach space $X$ if and only if $L^{\phi}(\mu, G)$ is a Chebyshev subspace of $L^{\phi}(\mu, X)$, where $L^{\phi}(\mu, X)$ is an Orlicz function space with Luxemburg norm.

Acknowledgement. The author would like to thank the referee for his/her suggestions.

\section{REFERENCES}

1. A.A.Hakawati and S.A.Dwaik, On best approximation in $L^{1}(\mu, X)$ and $L^{\phi}(\mu, X)$, Annals of Pure and Applied Mathematics, 12(1) (2016) 1-8 .

2. A.A.Hakawati and S.A.Dwaik. On best approximation in $L^{p}(\mu, X)$ and $L^{\phi}(\mu, X)$, $1 \leq p<\infty$, Journal of Mathematics and Informatics, 6 (2016) 31-39.

1. A.A.Hakawati and G.Ghawadrah, Proximinally additive chebychev spaces in $L^{\phi}(\mu, X)$ and $L^{p}(\mu, X), 1 \leq p<\infty$, Progress in Nonlinear Dynamics and Chaos, $4(2)$ (2016) 103-110 


\section{G. Ghawadrah}

2. W.Deeb and R.Khalil, Best approximation in $L(X, Y)$, Math. Proc. Cambridge Philos. Soc., 104 (1988) 527-531.

3. W.Deeb, Multipliers and isometries of Orlicz Spaces, In "Proceedings of the conference on Mathematical Analysis and its Applications, (Kuwait, 1985), Volume 3 of KFAS proc. ser. Pages 159-165. Perganon Oxford 1988.

4. I.Singer, Best Approximation In Normed Linear Spaces By Elements of Linear Subspaces, Springer-Verlay, NewYork.

5. W.Light and W.Cheney, Approximation Theory in Tensor Product Spaces, Lecture Notes in Math. 1169, Springer-Verlag Berlin, 9-155, 1985.

6. H.Al-Minawi and S.Ayesh, Best approximation in Orlicz spaces, International Journal of Mathematics and Mathematical Sciences, 14(2) (1991) 245-252.

7. J.Xu, Proximinality in Banach space valued Musielak-Orlicz spaces, Journal of Inequalities and Applications, 2014.1 (2014) 146. 